\title{
Optimal Boundary Control for Cascaded Parabolic PDEs with Constraints
}

\author{
Chao $\mathrm{HE}^{1, \mathrm{a}}$, Junmin $\mathrm{LI}^{1, \mathrm{~b}}$ \\ ${ }^{1}$ School of Mathematics and Statistics, Xidian University, Xi'an P.R. China \\ axidianhechao@126.com, ${ }^{\mathrm{b}}$ jmli@mail.xidian.edu.cn
}

Keywords: Optimal boundary control; Cascaded PDEs; Control constraints; Adjoint equation

\begin{abstract}
In this paper, the optimal boundary control problem in finite time horizon for linear parabolic partial differential equations cascaded through Robin boundary condition is addressed. Firstly, the well-posedness of the solution for the cascaded system is presented. Secondly, with the existence of the optimal control, the adjoint equation and the first-order sufficient and necessary condition are derived. Also, the well-posedness of the adjoint equation is guaranteed. Thirdly, to implement the optimal control, the optimal system consisting of a forward-in-time state equation and a backward-in-time adjoint equation coupled with the optimal boundary control is derived. Finally, a numerical example is presented to illustrate this method.
\end{abstract}

\section{Introduction}

Optimal control with partial differential equation (PDE) constrained has been more and more attracted in recent several decades, since its widely application in engineering. An earlier detailed study of the optimal control governed by PDEs can be discovered in [1]. Recently, with the effort in computation, the optimal control problem with PDEs becomes easier in implementation than before. Thus, a relatively amount of research results have been presented, for instance, the optimal control for parabolic PDEs and elliptic PDEs [2] or the infinite dimensional system optimal control [3], the optimal control for wave equation [4], etc. More recently, some special equation have been studied for the optimal control theory, such as the Cahn-Hilliard equation [5] [6], the viscous Dullin-Gottwalld-Holm [7], the viscous Degasperis-Procesi equation [8], etc. Although much attention has been paid on these optimal control problems, the optimal control for the cascaded PDEs remains to be studied.

Cascaded PDEs are commonly used in many practical problems, such as electromagnetic coupling, mechanical coupling, and coupled chemical reactions. It is meaningful to consider the optimal control for cascaded PDEs. Therefore, we first consider the optimal boundary control for the linear parabolic PDEs cascaded through the boundary. As well known, the optimal control for parabolic PDEs needs the specific well-posedness of the solutions since the existence of time variable. The well-posedness of the parabolic system was well studied in [2]. Due to the existence of the cascaded term, the well-posedness of cascaded system will become more complicated. In this paper, we first consider the well-posedness of the solution, which makes sense of the optimal control. Then, based on the optimization theory, the first-order sufficient and necessary optimality condition is obtained by using the Fréchet derivative and the continuity of the solution operators. Finally, a projected optimal control with the adjoint state is derived. Furthermore, we introduce the projected gradient method to get the control numerically. A numerical example is given to illustrate this method.

\section{PDE-constrained control problem}

We consider the following optimal control with linear quadratic functional: 


$$
\begin{aligned}
J\left(y_{1}, y_{2}, u\right) & =\frac{\lambda_{1}}{2} \iint_{Q_{1}}\left(y_{1}(x, t)-y_{d_{1}}(x, t)\right)^{2} d x d t+\frac{\lambda_{2}}{2} \iint_{Q_{2}}\left(y_{2}(x, t)-y_{d_{2}}(x, t)\right)^{2} d x d t \\
& +\frac{\lambda_{u}}{2} \iint_{\Sigma_{3}} u(x, t)^{2} d x d t,
\end{aligned}
$$

subject to

$$
\begin{array}{llll}
y_{1 t}-\Delta y_{1}+c_{1} y_{1}=0 & \text { in } Q_{1}=\Omega_{1} \times(0, T) & y_{2 t}-\Delta y_{2}+c_{2} y_{2}=0 & \text { in } Q_{2}=\Omega_{2} \times(0, T) \\
\partial_{v} y_{1}+\alpha_{1} y_{1}=\beta_{1} y_{2} & \text { in } \Sigma_{1}=\Gamma_{1} \times(0, T) & \partial_{v} y_{2}+\alpha_{2} y_{2}=\beta_{2} u & \text { in } \Sigma_{3}=\Gamma_{3} \times(0, T) \\
\partial_{v} y_{1}+\alpha_{1} y_{1}=b_{1} & \text { in } \Sigma_{2}=\Gamma_{2} \times(0, T) & \partial_{v} y_{2}+\alpha_{2} y_{2}=b_{2} & \text { in } \Sigma_{4}=\Gamma_{4} \times(0, T) \\
y_{1}(\cdot, 0)=y_{10}(x) & \text { in } \Omega_{1} & y_{2}(\cdot, 0)=y_{20}(x) & \text { in } \Omega_{2}
\end{array}
$$

(1.2)

with the control constraints:

$$
U_{a d}: u \in L^{2}\left(\Sigma_{3}\right): u_{a}(x, t) \leq u(x, t) \leq u_{b}(x, t) \text {, a.e. }(x, t) \in \Sigma_{3},
$$

where $\Omega_{1}, \Omega_{2} \in R^{N}$ are bounded Lipschitz domains with Lipschitz boundaries $\Gamma_{1} \cup \Gamma_{2}=\partial \Omega_{1}$, $\Gamma_{3} \cup \Gamma_{4}=\partial \Omega_{2}, \partial \Omega_{1} \cap \partial \Omega_{2}=\Gamma_{1}, T>0$ is a finite time constant and $c_{1}, c_{2}, \alpha_{1}, \alpha_{2}, \beta_{1}, \beta_{2}, b_{1}, b_{2}$, $y_{10}, y_{20}$ are functions which will be classified in the following Assumption 1. In this paper, we will design the controller signal $u(x, t)$ in Eq.1.3 to minimize the performance Eq.1.1 with respect to the reference signals $y_{d_{1}}(x, t) \in L^{2}\left(Q_{1}\right)$ and $y_{d_{2}}(x, t) \in L^{2}\left(Q_{2}\right)$.

Well-posedness of the solution. To consider the existence and regularity of the solution, we have the following assumption.

Assumption 1. Let $\Omega_{1}, \Omega_{2} \subset R^{N}$ be bounded Lipschitz domains with Lipschitz boundaries $\Gamma_{1} \cup \Gamma_{2}=\partial \Omega_{1}, \quad \Gamma_{3} \cup \Gamma_{4}=\partial \Omega_{2}, \partial \Omega_{1} \cap \partial \Omega_{2}=\Gamma_{1}$ and let $T>0$ denote a fixed final time. Moreover, assume that functions $c_{1} \in L^{\infty}\left(Q_{1}\right), c_{2} \in L^{\infty}\left(Q_{2}\right), \alpha_{1} \in L^{\infty}\left(\Sigma_{1} \cup \Sigma_{2}\right), \beta_{1} \in L^{\infty}\left(\Sigma_{1}\right), b_{1} \in L^{2}\left(\Sigma_{2}\right)$, $\alpha_{2} \in L^{\infty}\left(\Sigma_{3} \cup \Sigma_{4}\right), \beta_{2} \in L^{\infty}\left(\Sigma_{3}\right), b_{2} \in L^{2}\left(\Sigma_{4}\right), y_{10} \in L^{2}\left(\Omega_{1}\right), y_{20} \in L^{2}\left(\Omega_{2}\right)$, where $\alpha_{1}(x, t) \geq 0$ for almost every $(x, t) \in \Sigma_{1} \cup \Sigma_{2}$ and $\alpha_{2}(x, t) \geq 0$ for almost every $(x, t) \in \Sigma_{3} \cup \Sigma_{4}$ are prescribed.

The space $W_{2}^{1,0}(Q)$ denotes the normed space of all functions $y \in L^{2}(Q)$ having weak first-order partial derivatives with respect to $\left(x_{1}, x_{2}, \ldots, x_{N}\right)$ in $L^{2}(Q)$, endowed with the norm: $\|y\|_{W_{2}^{1,0}(Q)}=\left(\int_{0}^{T} \int_{\Omega}\left(|y(x, t)|^{2}+|\nabla y(x, t)|^{2}\right) d x d t\right)^{1 / 2}$.

From the Theorem 7.8 in [2] or [9], assume that assumption 1 holds. Then, the initial-boundary value problem Eq.1.2 has a unique solution $y_{1} \in W_{2}^{1,0}\left(Q_{1}\right), y_{2} \in W_{2}^{1,0}\left(Q_{2}\right)$. Furthermore, since the definition of weak solutions (variational equalities holding for $v_{1} \in W_{2}^{1,1}\left(Q_{1}\right), v_{2} \in W_{2}^{1,1}\left(Q_{1}\right)$ such that $v_{1}(\cdot, T)=0$ and $\left.v_{2}(\cdot, T)=0\right)$, the solutions $y_{1} \in W_{2}^{1,0}\left(Q_{1}\right)$ and $y_{2} \in W_{2}^{1,0}\left(Q_{2}\right)$ are difficult to consider the optimal control. Then, based on this solution space, we derive another solution space $W(0, T) \quad: \quad W_{1}(0, T)=\left\{y_{1} \in L^{2}\left(0, T ; H^{1}\left(\Omega_{1}\right)\right): y_{1 t} \in\left(0, T ; H^{1}\left(\Omega_{1}\right)^{*}\right)\right\} \quad$ and $W_{2}(0, T)=\left\{y_{2} \in L^{2}\left(0, T ; H^{1}\left(\Omega_{2}\right)\right): y_{2 t} \in\left(0, T ; H^{1}\left(\Omega_{2}\right)^{*}\right)\right\}$ with $H^{1}\left(\Omega_{i}\right)^{*}$ being the dual space of $H^{1}\left(\Omega_{i}\right)$. Then, we have the following theorem.

Theorem 1. Assume that Assumption 1 holds, the boundary control u belongs to $L^{2}\left(\Sigma_{3}\right)$. Then, the initial-boundary value problem Eq.1.2 has a unique solution $y_{1} \in W_{1}(0, T), y_{2} \in W_{2}(0, T)$. Besides, there exists some constants $c>0$ that does not depend on $\left(b_{1}, b_{2}, u, y_{10}, y_{20}\right)$ such that 


$$
\left\|y_{1}\right\|_{W_{1}(0, T)}+\left\|y_{2}\right\|_{W_{2}(0, T)} \leq c\left(\left\|b_{1}\right\|_{L^{2}\left(\Sigma_{2}\right)}+\left\|b_{2}\right\|_{L^{2}\left(\Sigma_{4}\right)}+\left\|y_{10}\right\|_{L^{2}\left(\Omega_{1}\right)}+\left\|y_{20}\right\|_{L^{2}\left(\Omega_{2}\right)}+\|u\|_{L^{2}\left(\Sigma_{3}\right)}\right) .
$$

Proof. To proof the theorem 1, one way is to follow the proof in [1]. Another way is based on the solution space $W_{2}^{1,0}\left(Q_{1}\right), W_{2}^{1,0}\left(Q_{2}\right)$. Firstly, consider the $y_{2}$ system. Since the assumption in theorem 1, we have $y_{1}=W_{2}^{1,0}\left(Q_{1}\right)$ and $y_{2} \in W_{2}^{1,0}\left(Q_{2}\right)$. Then, from the theorem 3.12 in [2], we have the $y_{2} \in W_{2}(0, T)$. With the cascaded term $\left.y_{2}\right|_{\Sigma_{1}}$ belongs to $L^{2}\left(\Sigma_{1}\right)$. Based on the proof of theorem 3.12 in [2], we have conclude that $y_{1} \in W_{1}(0, T)$. Similarly, the estimate of the form Eq.2 can be obtained directly from Theorem 3.13 in [2].

Since $y_{1} \in W_{1}(0, T)$ and $y_{2} \in W_{2}(0, T)$, from the theorem 3.10 in [2], with the continuous embedding: $W_{1}(0, T) \rightarrow C\left([0, T], H^{1}\left(\Omega_{1}\right)\right)$ and $W_{2}(0, T) \rightarrow C\left([0, T], H^{1}\left(\Omega_{2}\right)\right)$, we conclude that $y_{1} \in C\left([0, T], H^{1}\left(\Omega_{1}\right)\right)$ and $y_{2} \in C\left([0, T], H^{1}\left(\Omega_{2}\right)\right)$. Then, the initial and final values $y_{1}(0), y_{1}(T)$, $y_{2}(0)$ and $y_{2}(T)$ are meaningful in spaces $H^{1}\left(\Omega_{i}\right), i=1,2$. Then, the adjoint equation of the state equation Eq.1.2 is meaningful. In the following, we will consider the optimal boundary control for the cascaded system Eq.1.2 with performance index Eq. 1.1.

\section{First-order necessary optimality condition}

From theorem 1, the solution in $y_{1} \in W_{1}(0, T)$ and $y_{2} \in W_{2}(0, T)$ can be represented of the form $y_{2}=G_{\Sigma_{3}} \beta_{2} u+G_{\Sigma_{4}} b_{2}+G_{20} y_{20}=S_{2} u+b_{2}^{*} \quad, \quad y_{1}=G_{\Sigma_{1}} \beta_{1} \tau_{\Sigma_{1}} S_{2} u+G_{\Sigma_{1}} \beta_{1} \tau_{\Sigma_{1}} b_{2}^{*}+G_{\Sigma_{2}} b_{1}+G_{10} y_{10}=S_{1} u+b_{1}^{*}$, with continuous linear solution operators $G_{\Sigma_{3}}: L^{2}\left(\Sigma_{3}\right) \rightarrow W_{2}(0, T)$ for $b_{2}=0, y_{20}=0$, $G_{\Sigma_{4}}: L^{2}\left(\Sigma_{4}\right) \rightarrow W_{2}(0, T)$ for $\beta_{2} u=0, y_{20}=0 \quad, \quad G_{20}: L^{2}\left(\Omega_{2}\right) \rightarrow W_{2}(0, T)$ for $b_{2}=0, \beta_{2} u=0$, $G_{\Sigma_{1}}: L^{2}\left(\Sigma_{1}\right) \rightarrow W_{1}(0, T)$ for $b_{1}=0, y_{10}=0, \quad G_{\Sigma_{2}}: L^{2}\left(\Sigma_{2}\right) \rightarrow W_{1}(0, T)$ for $\beta_{1} y_{2}=0, y_{10}=0$ and $G_{10}: L^{2}\left(\Omega_{1}\right) \rightarrow W_{1}(0, T)$ for $b_{1}=0, \beta_{1} y_{2}=0$, where $\tau_{\Sigma_{1}}$ be the trace operator of $y_{2}$ on the boundary $\Sigma_{1}, b_{1}^{*}$ and $b_{2}^{*}$ are fixed through the given $b_{1}, b_{2}, y_{10}$ and $y_{20}$.

Due to theorem 1 , we have known that the solution $y_{1} \in W_{1}(0, T)$ and $y_{2} \in W_{2}(0, T)$. Since the trace theorem in [10], $y_{1} \in L^{2}\left(0, T ; H^{1}\left(\Omega_{1}\right)\right) \subset L^{2}\left(Q_{1}\right)$ and $y_{2} \in L^{2}\left(0, T ; H^{1}\left(\Omega_{2}\right)\right) \subset L^{2}\left(Q_{2}\right)$. Therefore, the optimal boundary control for index $J\left(y_{1}, y_{2}, u\right)$ is meaningful. Furthermore, due to the admissible control set $U_{a d}$ is a nonempty, closed, bounded, convex subset $U_{a d} \subset L^{2}\left(\Sigma_{3}\right)$, where $L^{2}\left(\Sigma_{3}\right)$ is a reflexive space. Simultaneously, with the linear continuous solution operators of Eq.1.2, the coefficient is chosen as $\lambda_{u}>0$. Then, from theorem 3.15 in [2], the linear quadratic optimal control problem Eq.1.1-Eq.1.3 admits the unique optimal boundary control. What's more, since the linear form of system Eq.1.2 and the convexity of $J$, it is easily seen that the first-order necessary condition is also the sufficient optimality condition.

Furthermore, we reduce the optimal control problem as $\hat{J}(u)=J\left(S_{1} u+b_{1}^{*}, S_{2} u+b_{2}^{*}, u\right)$. Then, by the Fréchet differentiability of $\hat{J}(u)$, we have the variational inequality

$$
\begin{aligned}
\hat{J}^{\prime}(\bar{u})(u-\bar{u}) & =\lambda_{1} \iint_{Q_{1}}\left(\bar{y}_{1}-y_{d_{1}}\right) S_{1}(u-\bar{u}) d x d t+\lambda_{2} \iint_{Q_{2}}\left(\bar{y}_{2}-y_{d_{2}}\right) S_{2}(u-\bar{u}) d x d t \\
& +\lambda_{u} \iint_{\Sigma_{3}} \bar{u}(u-\bar{u}) d s d t \geq 0
\end{aligned}
$$

In Eq.3, $\bar{y}_{1}, \bar{y}_{2}$ and $\bar{u}$ denote the optimal state and control, respectively. Let $y_{1}=S_{1}(u-\bar{u})$ and $y_{2}=S_{2}(u-\bar{u})$. Since the solution operator, we have $y_{1}=S_{1}(u-\bar{u})=G_{\Sigma_{1}} \beta_{1} \tau_{\Sigma_{1}} y_{2}$. To deal with Eq.3 
further, we introduce a lemma about the adjoint equation as follows.

Lemma 1 (adjoint equation) For the optimal control problem Eq.1.1-Eq.1.3, from the induced variational inequality Eq.3, consider subsystems $y_{1}=S_{1}(u-\bar{u})$ and $y_{2}=S_{2}(u-\bar{u})$, the adjoint equation for system Eq.1.2 is given as

$$
\begin{array}{llll}
-p_{2 t}-\Delta p_{2}+c_{2} p_{2}=\lambda_{2}\left(\bar{y}_{2}-y_{d 2}\right) & Q_{2} & \\
\partial_{v} p_{2}+\alpha_{2} p_{2}=\lambda_{1} \beta_{1} p_{1} & \Sigma_{1} & -p_{1 t}-\Delta p_{1}+c_{1} p_{1}=\bar{y}_{1}-y_{d 1} & Q_{1} \\
\partial_{v} p_{2}+\alpha_{2} p_{2}=0 & \partial \Omega_{2} / \Gamma_{1} \times(0, T) & \partial_{v} p_{1}+\alpha_{1} p_{1}=0 & \partial \Omega_{1} \times(0, T) \\
p_{2}(\cdot, T)=0 & \Omega_{2} & p_{1}(\cdot, T)=0 & \Omega_{1}
\end{array}
$$

and $p_{1} \in W_{1}(0, T), p_{2} \in W_{2}(0, T)$.

Proof. Combing with theorem 3.18 in [2], one has

$$
\lambda_{1} \iint_{Q_{1}}\left(\bar{y}_{1}-y_{d_{1}}\right) S_{1}(u-\bar{u}) d x d t=\lambda_{1} \iint_{\Sigma_{1}} p_{1} \beta_{1} S_{2}(u-\bar{u}) d s d t
$$

with the adjoint $p_{1}$ subsystem as Eq.4. Since Eq.5, Eq.3 can be written as

$$
\begin{aligned}
\lambda_{1} \iint_{Q_{1}}\left(\bar{y}_{1}-y_{d_{1}}\right) S_{1}(u-\bar{u}) d x d t & +\lambda_{2} \iint_{Q_{2}}\left(\bar{y}_{2}-y_{d_{2}}\right) S_{2}(u-\bar{u}) d x d t \\
& =\lambda_{1} \iint_{\Sigma_{1}} p_{1} \beta_{1} S_{2}(u-\bar{u}) d s d t+\lambda_{2} \iint_{Q_{2}}\left(\bar{y}_{2}-y_{d_{2}}\right) S_{2}(u-\bar{u}) d x d t .
\end{aligned}
$$

Then, consider the subsystem $y_{2}=S_{2}(u-\bar{u})$, by using theorem 3.18 in [2], it is easily seen that

$$
\lambda_{1} \iint_{\Sigma_{1}} p_{1} \beta_{1} S_{2}(u-\bar{u}) d s d t+\lambda_{2} \iint_{Q_{2}}\left(\bar{y}_{2}-y_{d_{2}}\right) S_{2}(u-\bar{u}) d x d t=\iint_{\Sigma_{3}} \beta_{2} p_{2}(u-\bar{u}) d s d t,
$$

with the adjoint state $p_{2}$ satisfying Eq.4. Above all, we have obtained the adjoint equation Eq.4, where the well-posedness can be considered with the time transform as: $\tau=t-T$. Then system Eq.4 can be transformed the general forward in time parabolic equation as Eq.1.2. From theorem 1, we have $p_{1} \in W_{1}(0, T), p_{2} \in W_{2}(0, T)$.

Theorem 2. (Sufficient and necessary optimality condition) With the assumption 1 holding, the sufficient and necessary optimality condition of optimal boundary control problem is $\iint_{\Sigma_{3}}\left(\beta_{2} p_{2}+\lambda_{u} \bar{u}\right)(u-\bar{u}) d s d t \geq 0, \quad \forall u \in U_{a d}$. Furthermore, the optimal control can be represented as $\bar{u}(x, t)=\mathrm{P}_{\left[u_{a}(x, t), u_{b}(x, t)\right]}\left(-\left.\frac{1}{\lambda_{u}} \beta_{2} p_{2}\right|_{\Sigma_{3}}\right)$ with $\mathrm{P}_{\left[u_{a}(x, t), u_{b}(x, t)\right]}$ being the projection of $R$ onto $\left[u_{a}(x, t) u_{b}(x, t)\right]$ pointwise on $\Sigma_{3}: \mathrm{P}_{\left[u_{a}(x, t), u_{b}(x, t)\right]}(\cdot):=\min \left\{u_{b}(x, t), \max \left\{u_{a}(x, t), \cdot\right\}\right\}$.

Proof. The variational inequality Eq.3 can be formulated as the weak minimum principle pointwise. That is $\left(\left.\beta_{2} p_{2}\right|_{\Sigma_{3}}+\lambda_{u} \bar{u}\right)(u-\bar{u}) \geq 0, \quad \forall u \in\left[u_{a}(x, t), u_{b}(x, t)\right]$, which is equivalent to the minimum principle $\left.\beta_{2} p_{2}\right|_{\Sigma_{3}} \bar{u}+\frac{\lambda_{u}}{2} \bar{u}^{2}=\min _{u \in\left[u_{a}(x, t), u_{b}(x, t)\right]}\left\{\left.\beta_{2} p_{2}\right|_{\Sigma_{3}} u+\frac{\lambda_{u}}{2} u^{2}\right\}$. Obviously, we obtain the optimal control with $\lambda_{u}>0$.

\section{Numerical example}

To illustrate the optimal control as the adjoint equation Eq.4 and optimal control, we consider the system Eq.1.2 over the domain $\Omega_{1}=\Omega_{2}=[0,1] \times(0, T)$, with coefficients $c_{1}=0, c_{2}=0.4, \alpha_{1}=0.13$, $\alpha_{2}=0.4, \beta_{1}=1.0, \beta_{2}=1.0, b_{1}(t)=0.3, b_{2}(t)=0.2$. Besides, the initial state are given as $y_{10}(x)=0.2 \sin x, y_{20}(x)=1-x^{2}$. Fixed time constant $T=5.0$ and reference trajectories are given as $y_{d 1}(x, t)=0.7, \forall(x, t) \in \Omega_{1}$ and $y_{d 2}(x, t)=0, \forall(x, t) \in \Omega_{2}$. The regulation coefficients are 
chosen as $\lambda_{1}=4.0, \lambda_{2}=0.001, \lambda_{u}=0.0005$. Finally, the control constraint is given as $u_{a}=-0.6$ and $u_{b}=0.0$. Then, the adjoint equation Eq.1.2 and theorem 2, the optimal control is $\bar{u}(x, t)=\mathrm{P}_{\left[u_{a}, u_{b}\right]}\left(-\frac{1}{\lambda_{u}} p_{2}(1, t)\right)$. To obtain the optimal control and state, we use the projected gradient methods, whose convergence analysis can refer to [11].

Initialize: Choose any initial control $u_{a} \leq u_{0}(t) \leq u_{b}$. Step1: Solve the state system Eq.1.2 with control $u_{n}(t)$, we obtain the state $y_{1 n}, y_{2 n}$. Step2: Based on the $y_{1 n}, y_{2 n}$, we solve the adjoint equation Eq.4, we get the adjoint state $p_{1 n}, p_{2 n}$. Then, following the variational inequality in theorem 2, we obtain the negative gradient: $v_{n}(t)=-\hat{J}^{\prime}\left(u_{n}\right)=-\left(p_{2 n}(1, t)+\lambda_{u} u_{n}\right)$. Step3: Determine the optimal step size $s_{n}: \hat{J}\left(\mathrm{P}_{\left[u_{a}, u_{b}\right]}\left\{u_{n}+s_{n} v_{n}\right\}\right)=\min _{s>0} \hat{J}\left(\mathrm{P}_{\left[u_{a}, u_{b}\right]}\left\{u_{n}+s v_{n}\right\}\right)$. Step4: Let $u_{n+1}=\mathrm{P}_{\left[u_{a}, u_{b}\right]}\left\{u_{n}+s_{n} v_{n}\right\}$, go to Step 1 .

Following the projected gradient method, we obtain the optimal performance index after 15 iterations: $\hat{J}\left(u_{15}\right)=0.2042$. Fig. 1 (a) shows the curve of the performance index $\hat{J}\left(u_{n}\right)$ with respect to the iteration $n$. The variation of the control $u_{n}(t)$ with respect to iteration $n$ is given in Fig. 1 (b).
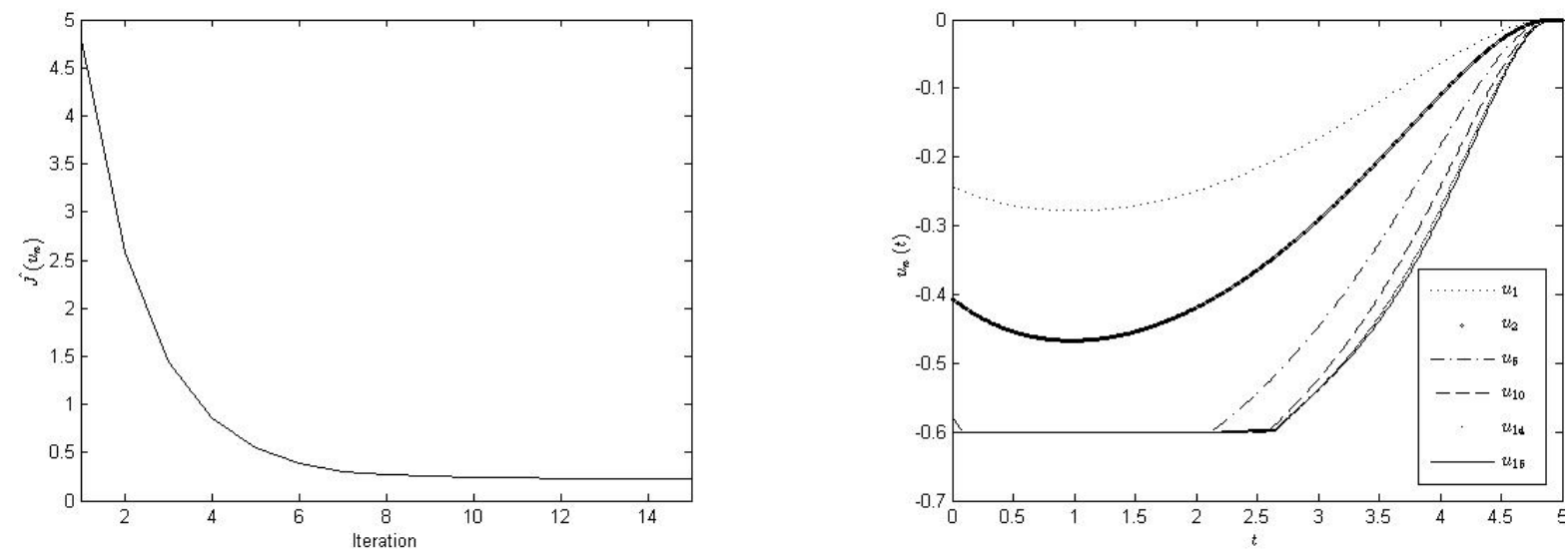

(a) The curve of the performance index vs. iteration $\quad$ (b) The variation of the control vs. iteration Fig.1. Numerical results

\section{Conclusion}

In this paper, the optimal boundary control for linear parabolic cascaded system has been addressed. Based on the well-posedness analysis, combined with adjoint equation, the first-order sufficient and necessary optimality condition has been derived. Then, a projected control scheme has been obtained. Finally, to illustrate the optimality system, a numerical example has been solved by the projected gradient method.

\section{Acknowledgement}

In this paper, the research was supported in part by Ph.D. Programs Foundation of Ministry of Education of China under Grant 20130203110021 and in part by NNSF of China No. 61573013.

\section{References}

[1]Jacques-Louis Lions. Optimal Control of Systems Governed by Partial Differential Equations[M]. Springer, Berlin,1971. 
[2]Fredi Tröltzsch. Optimal Control of Partial Differential Equations: Theory, Methods and Applications[M]. American Mathematical Society, Providence, 2010.

[3] Xungjing Li and Jiongmin Yong. Optimal Control Theory for Infinite Dimensional Systems[M]. Birkhäuser, Boston, 1995.

[4]Martin Gugat, Emmanuel Trélat, and Enrique Zuazua. Optimal Neumann control for the 1d wave equation: Finite horizon, infinite horizon, boundary tracking terms and the turnpike property[J]. Systems \& Control Letters, 2016, 90, 61-70.

[5]Jiongmin Yong and Songmu Zheng. Feedback stabilization and optimal control for the Cahn-Hilliard equation[J]. Nonlinear Analysis, 1991, 17(5):431-444.

[6]Xiaopeng Zhao and Changchun Liu. Optimal control problem for viscous Cahn-Hilliard equation[J]. Nonlinear Analysis: Theory Methods \& Applications, 2011, 74(17):6348-6357.

[7]Chunyu Shen, Lixin Tian, and Anna Gao. Optimal control of the viscous Dullin-Gottwalld-Holm equation[J]. Nonlinear Analysis: Real World Applications, 2010, 11(1):480-491.

[8]Lixin Tian and Chunyu Shen. Optimal control of the viscous Degasperis-Procesi equation[J]. Journal of Mathematical Physics, 2007, 48, 113513.

[9]O. A. Ladyenskaja, V. A. Solonnikov, and N. N. Uralceva. Linear and Quasilinear Equations of Parabolic Type[M]. American Mathematical Society, Providence, 1968.

[10]Robert A. Adams. Sobolev Space[M]. Boston, Academic Press, 1978.

[11]Michael Hinze, Rene Pinnau, Michael Ulbrich, Stefan Ulbrich. Optimization with PDE constraints, Mathematical Modelling: Theory and Applications[M]. Springer, Berlin, 2009. 